\title{
MUSIQUE ET MIGRATION : LE CARNAVAL DE COLOGNE COMME ÉTAT D'ESPRIT
}

\author{
Birgit Ellinghaus et Monika Salzbrunn
}

Université de Poitiers | «Revue européenne des migrations internationales »

2019/3 Vol. 35 | pages 33 à 40

ISSN 0765-0752

Article disponible en ligne à l'adresse :

https://www.cairn.info/revue-europeenne-des-migrationsinternationales-2019-3-page-33.htm

Distribution électronique Cairn.info pour Université de Poitiers.

(C) Université de Poitiers. Tous droits réservés pour tous pays.

La reproduction ou représentation de cet article, notamment par photocopie, n'est autorisée que dans les limites des conditions générales d'utilisation du site ou, le cas échéant, des conditions générales de la licence souscrite par votre établissement. Toute autre reproduction ou représentation, en tout ou partie, sous quelque forme et de quelque manière que ce soit, est interdite sauf accord préalable et écrit de l'éditeur, en dehors des cas prévus par la législation en vigueur en France. Il est précisé que son stockage dans une base de données est également interdit. 


\section{Revue européenne des migrations}

internationales

vol. 35 - $n^{\circ} 3$ et $4 \mid 2019$

Danses, musiques et (trans)nationalismes

\section{Musique et migration : le carnaval de Cologne comme état d'esprit}

Music and Migration: The Cologne Carnival as a Way of Life

Música y migración: el carnaval de Colonia en tanto modo de vida

Birgit Ellinghaus et Monika Salzbrunn

\section{(2) OpenEdition}

\section{Journals}

Édition électronique

URL : http://journals.openedition.org/remi/13289

ISSN : 1777-5418

Éditeur

Université de Poitiers

Édition imprimée

Date de publication : 1 décembre 2019

Pagination : 33-40

ISBN : 979-10-90426-65-8

ISSN : 0765-0752

Distribution électronique Cairn

\section{CAIRN I INFO}

CHERCHER, REPÉRER, AVANCER

\section{Référence électronique}

Birgit Ellinghaus et Monika Salzbrunn, « Musique et migration : le carnaval de Cologne comme état d'esprit », Revue européenne des migrations internationales [En ligne], vol. 35 - n³ et 4 | 2019, mis en ligne le 01 mars 2020, consulté le 20 avril 2020. URL : http://journals.openedition.org/remi/13289 


\title{
Chronique d'actualité
}

\section{Musique et migration : le carnaval de Cologne comme état d'esprit}

\author{
Birgit Ellinghaus ${ }^{1}$ et Monika Salzbrunn²
}

Cité d'immigration vieille de 2000 ans, Cologne et sa vie festive sont marquées par des courants musicaux des plus divers. Le carnaval, qui se revendique entre autres de I'héritage du culte voué à Dionysos, et dont la première évocation écrite dans les documents de la ville date de 1341, est caractérisé par la moquerie des autorités militaires (qui ont interdit la fête pendant l'occupation de la ville par la Prusse) et le blasphème à l'encontre des porteurs de I'autorité catholique (Fuchs et al., 1972), souvent exprimé par des chansons et des parodies. Institution emblématique du multiculturalisme colonais, le carnaval réunit une scène musicale hétéroclite, et résonne avec une histoire de rencontres et de passages.

\section{Cologne : ville libre et carrefour de rencontres}

Le nom "Köln » (fr. "Cologne ", it. "Colonia ") est révélateur des origines de cette ville qui compte aujourd'hui plus d'un million d'habitants. Fondée en 50 par Agrippine la Jeune comme colonie romaine du Nord et située au bord du Rhin, Cologne représente depuis 2000 ans la porte et les oreilles du monde : c'est une ville de passage et de rencontres, d'abord pour les légionnaires, puis pour les soldats, les commerçants et, depuis le Moyen Âge, pour les pèlerins, enfin pour les touristes qui y ont parfois laissé leur marque (comme I' "Eau de Cologne " créée par la famille Farina arrivée au XVIIle siècle à Cologne !). Elle a été la plupart du temps une ville libre gérée par des habitant·e.s. Depuis sa fondation, elle n'a été qu'à de rares occasions sous domination étrangère, comme, par exemple, lors de l'occupation par les troupes de Napoléon. L'Église catholique - bien que contestée par une frange de la population - y a joué un rôle important, autant sur le plan politique, économique, culturel que moral, dans la vie quotidienne des habitants. Cela est encore perceptible aujourd'hui dans le paysage urbain,

\footnotetext{
1 Directrice, alba KULTUR, Zwirnerstr. 26, D - 50678 Cologne, Allemagne ; birgit@albakultur.de

2 Sociologue et anthropologue, Professeure de Religions, Migration, Diasporas et Directrice du projet ERC ARTIVISM, Université de Lausanne, Anthropole 5067, 1015 Lausanne, Suisse; monika.salzbrunn@unil.ch

Le projet ERC ARTIVISM (www.erc-artivism.ch) a reçu un financement du Conseil européen de la recherche (European Research Council - ERC) dans le cadre du programme de recherche et d'innovation de I'Union européenne "Horizon 2020 " (ARTIVISM - convention de subvention No 681880).
} 
avec la cathédrale et douze imposantes églises romanes, ainsi qu'au niveau du paysage culturel : I'Église locale, ayant une base très critique envers le Vatican, pratique la plupart du temps un "catholicisme rhénan ", libéral, et s'engage en tant qu'organisateur et bailleur de fonds. D'un autre côté, la " Freie Szene ", une large scène musicale " libre " comportant tous les styles et ne bénéficiant que de maigres subventions publiques par rapport à I'Opéra et la Philharmonie, est le cœur battant d'une création musicale particulièrement dynamique.

La ville, qui promeut fièrement ses origines multiculturelles ${ }^{3}, \mathrm{n}^{\prime} \mathrm{a}$ pourtant pas été épargnée par l'Histoire : elle a été inondée à plusieurs reprises, brûlée lors de guerres, et presque complètement détruite par les bombardements de la Seconde Guerre mondiale. Dernière calamité en date : en 2009, des documents importants de I'histoire musicale ont été perdus dans l'effondrement des archives municipales de Cologne, à la suite d'erreurs de construction d'une ligne de métro, aggravées par un cas de corruption. Ceci illustre une autre facette de la réputation de Cologne considérée grâce à sa culture festive comme la ville la plus italienne au nord des Alpes : le "Klüngel ", une culture d'entraide marquée par des conflits d'intérêt.

Forte de cette histoire, Cologne est une des villes d'Allemagne incarnant une forte et ancienne union entre les traditions musicales allant de l'époque romaine jusqu'à nos jours, et la culture musicale locale, marquée par le dialecte. La ville a toujours abrité des milliers de musicien-ne.s amateurs et de professionnel-le.s de la musique d'origines diverses qui, encore aujourd'hui, se rapportent activement aux diverses sources de l'histoire de la musique de Cologne.

\section{Des sources historiques multiples}

Les musicien-ne's ont pour références des images mythiques de musiciens et d'instruments de l'époque romaine, très présentes dans les musées, les églises ainsi que dans la rue, comme la fameuse mosaïque dédiée à Dionysos dans le musée germano-romain à côté de la cathédrale.

II y a aussi toutes les histoires légendaires du peuple des "Veedel " (les quartiers populaires, premières destinations des immigré.e.s de travail), qui ont produit une poésie populaire particulière, accompagnée de chansons profondes, critiques et humoristiques. Le Kölsch, dialecte typique de Cologne et langue du peuple, joue ici un rôle important. II a intégré de nombreux éléments de la diversité linguistique qui a fait la richesse de la ville à toutes les époques, en premier lieu grâce à la langue française, transmise d'abord et surtout à travers les élites aux XVle et XVIle siècles et ensuite par les 12000 soldats révolutionnaires puis napoléoniens présents dans la ville (1794-1814) (Cornelissen, 2019). Le Kölsch est caractérisé par une intonation spécifique, considérée comme chantante et rhénane. II est utilisé comme une rébellion éloquente contre les

3 Le site officiel de la ville conclut le résumé de son histoire romaine par le constat "Déjà à l'époque, Cologne était multiculti " (https://www.stadt-koeln.de/politik-undverwaltung/presse/agrippina-kaiserin-aus-koeln). De plus, il y a vingt ans, I'office de tourisme lançait une campagne intitulée "Nos premiers Colonais étaient des étrangers " (Traductions par Monika Salzbrunn). 
autorités et comme une forme de résistance faite d'humour concis. Le Krätzcher en est l'incarnation musicale.

À l'origine, le Krätzcher (littéralement : " gratter ", métaphoriquement : " provoquer ") était une forme de poésie satirique en dialecte Kölsch. Ces poèmes ont été repris à la fin du XVIIle siècle par les ménestrels de la Rhénanie, chantés et orchestrés avec modération. Les chants Krätzcher sont aujourd'hui I'une des plus anciennes formes de musique traditionnelle orale en Rhénanie. Au cours des deux guerres mondiales, le Krätzcher a atteint de nouveaux sommets en tant que format critique et humoristique, exprimant avec peu de moyens ce que le peuple n'osait pas dire ouvertement. L'accompagnement instrumental des chansons était généralement simple, mais les paroles, souvent polysémiques, reflétaient de façon laconique une certaine philosophie et donnaient une raison de sourire et de réfléchir.

Contrairement à de nombreuses traditions de musique populaire, le Krätzcher n'a pas été instrumentalisé par les nazis. Au contraire, il a constitué une expression de résistance. Après la Seconde Guerre mondiale, le Krätzcher a presque complètement disparu de la scène. Ces chants n'étaient pas tabous dans l'Allemagne d'après-guerre, mais plutôt démodés, parce qu'ils ne répondaient pas au besoin d'harmonie et à la nécessité de vivre dans l'illusion d'un heile Welt (traduisible par " monde sain et réconfortant ") pendant les années de miracle économique ("Wirtschaftswunderjahre ").

Ce n'est que dans les années 1980 que le Krätzcher a connu une renaissance : le plus important groupe de rock en dialecte kölsch, Bläck Fööss, en a été l'étincelle initiale, car les musiciens ont inclus le Krätzcher dans leur répertoire. Non seulement le public a été sensibilisé à cette ancienne forme de chanson de Cologne, mais elle a aussi inspiré de nombreux autres artistes. À l'heure actuelle, le Krätzcher fait à nouveau partie du répertoire de la culture musicale locale. Parmi les représentants les plus importants de cette tradition, on compte notamment des rappeurs turcs, brésiliens et nigérians résidant à Cologne. Le dialecte kölsch est ainsi (re)devenu la carte d'identité de la ville et l'élément représentatif de la scène musicale locale. Lors d'un spectacle carnavalesque conçu "depuis la perspective des nouveaux arrivants " et prenant la forme d'un cabaret politique " entre les cultures ", la "Immi-Sitzung ", les nouveaux "Krätzcher " sont accompagnés de courants musicaux les plus divers. La devise des artistes est " Jede Jeck is von woanders " ("Chaque fêtard vient d'ailleurs ") $)^{4}$, un détournement du bon mot "Jede Jeck is anders " ("Chaque fêtard est différent "). Les deux devises incarnent en réalité l'ouverture d'esprit de la ville et la prise de conscience que tou-te-s ses résident·e-s sont des (enfants $d^{\prime}$ ) immigré·e.s.

Ces sources telles que le Krätzcher sont les bases d'un biotope musical et d'un son urbain propres à la ville de Cologne, qui s'expriment dans une variété de formes musicales, et dans le carnaval, institution emblématique et fédératrice.

4 Ces passages entre guillemets proviennent tous du site https://www.immisitzung.de/ index.php et ont été traduits par Monika Salzbrunn. 


\section{Le carnaval : une déclaration politique pour l'ouverture vers les autres}

La valeur particulière du carnaval de Cologne est qu'il s'agit d'une fête cathartique qui rassemble chaque année dans son biotope musical tous les groupes sociaux et tous les genres musicaux, et attire plus d'1 million de visiteurs à chaque édition. II représente le temps de l'année où les règles de vie sont mises au défi, où les gens se retrouvent dans les fêtes, où le nouveau se crée. En 2015, I'UNESCO a inscrit le carnaval de Cologne sur la Liste nationale du patrimoine culturel immatériel :

"Le carnaval transmet des sentiments de joie et donne la sensation de faire partie d'une communauté forte. Surtout en temps de bouleversement social, son pouvoir d'intégration est prouvé : la fête a donné un bel élan dans la période de la reconstruction de la ville après-guerre. La culture de l'accueil du carnaval est très forte. Les migrants trouvent un accès facile à la communauté régionale. Au sein de la communauté "jeck" [folle], se déguiser pour se glisser dans d'autres rôles, participer à des célébrations sauvages fait autant partie du carnaval que l'engagement volontaire et social. L'histoire de la fête du carnaval en Rhénanie remonte au début du XIIle siècle. Elle est définie comme une étape temporelle avant le carême dans la période de l'année chrétienne. [...] Au début du XIXe siècle, à Cologne, s'est développée une forme profane du carnaval. Depuis cette époque, le lundi du carnaval, un défilé masqué imite et caricature les grandes parades militaires. $n^{5}$

Dans le carnaval, on peut repérer d'autres spécialités musicales de Cologne : le Schunkeln, les fanfares de cuivre, les marching bands et les ensembles de tambours. Dans les défilés, la musique festive provient principalement de marches militaires, jouées par des fanfares et des ensembles de tambours de la banlieue ou des villages qui entourent Cologne, mais aussi des groupes de samba et batucada. Ces groupes se déplacent spontanément de bar en bar et accompagnent les défilés dans les quartiers. Dans les bals traditionnels donnés dans des salles et des tentes, on entend surtout une forme particulière de valse. En effet, il n'y a pas de piste de danse, et comme les personnes ne peuvent s'extraire des rangées de sièges, elles ont développé une danse qu'on peut effectuer tout en restant assis : le "Schunkeln ". Les spectateurs sont alors assis sur des bancs autour de longues tables et regardent de grands orchestres mener un programme de célébration joyeuse. On accroche ses bras à ceux des personnes assises à droite et à gauche et on se balance ensemble tout en chantant des chansons drôles. Lors de ces bals traditionnels, l'origine des carnavaleux ne joue aucun rôle. II ne s'agit ni d'une assimilation ni d'une intégration active des migrants. La devise est "Chaque Jeck est différent ", ce qui incarne la valeur de I'altérité, peu importe d'où elle provient. En 2008-2009, plusieurs monuments emblématiques de Cologne ont été représentés comme des personnes en train de "schunkeln ", avec des bras imbriqués, sur la médaille officielle d'un des quartiers les plus importants de Cologne : la nouvelle mosquée de Cologne, une des plus grandes et plus visibles d'Europe, y était représentée (dix ans avant son inauguration officielle !) à côté de la cathédrale, de la tour de télévision et du monument local Heliosturm.

5 Traduction en français du site https://www.unesco.de/kultur-und-natur/immaterielleskulturerbe/immaterielles-kulturerbe-deutschland/rheinischer-karneval 
À Cologne, le carnaval est de la sorte un état d'esprit, une déclaration politique pour l'ouverture vers les autres, incarnée par la diversité musicale et par une forme $d^{\prime}$ anarchisme et de transgression permanente ${ }^{6}$. Autour de ce carnaval, germent et évoluent une variété de formes musicales et de valorisations sonores du multiculturalisme.

\section{Un biotope multiculturel inspirant des expressions musicales variées}

La scène musicale de Cologne, comme celles d'autres villes, comporte une grande pluralité de genres musicaux, tels que le jazz et les musiques improvisées, le rock et la pop, la musique baroque, la musique contemporaine d'avantgarde, l'électronique, la musique classique et les musiques traditionnelles et du monde. Mais chaque genre de musique utilise le dialecte kölsch pour des créations actuelles. Les libres penseurs et les révoltés s'expriment en dialecte dans leurs hymnes contre le racisme et la xénophobie, qui trouve son apogée récent dans le mouvement "Arsch huh ". Ce dernier - littéralement : "lève ton cul ", métaphoriquement : " bouge-toi " contre le racisme et l'extrême droite - est né en 1992 après les attentats contre des réfugiés à Hoyerswerda en ex-RDA - et ses héros locaux et internationaux, BAP, Bläck Fööss, Brings ou Höhner - et il déplace les foules depuis 25 ans. Unis au-delà de leurs querelles au moment où le racisme émergeait pour la première fois sous forme d'une série d'attentats xénophobes meurtriers depuis la fin de la seconde guerre mondiale en Allemagne, ces groupes de musique protagonistes du carnaval et/ou groupes rock chantant en kölsch, avaient réuni 100000 spectateurs le 9 novembre 1992 au centre-ville afin de prendre position contre le racisme. En ce jour anniversaire de la " nuit de cristal " de 1938 (un pogrom contre la population juive), I'hymne "Arsch huh, Zäng ussenander " pour le vivre ensemble et le courage civil a été chanté pour la première fois. La chanson avait été écrite en quelques jours, en réaction aux attentats, par Vassilios "Nick " Nikitakis et Wolfgang Niedecken, chanteur du groupe BAP mondialement connu. Jean Jülich, un des résistants membres des "Edelweisspiraten " participait également à l'événement. Ce lien symbolique et personnel entre des protagonistes du carnaval, des résistants de la Seconde Guerre mondiale et des musicien.ne-s issu.e.s de la migration est récurrent, comme les exemples de "Humba " et du "Zigeunerfestival " le démontrent.

\section{Affirmer le multiculturel par la « world-music " : " Humba \& Famille "}

L'idée principale de la plateforme "Humba " ${ }^{7}$ est depuis le début des années 1990 d'affirmer la diversité musicale au cœur de la tradition populaire, collective, vivante et sonore de Cologne. Cette multiculturalité s'exprime dans

6 La vingtaine de marchés " officiels " et " alternatifs " de Noël incarne également cet esprit carnavalesque : plusieurs marchés gays au décor clinquant, un marché-Afterwork organisé par un Hollandais sur un terrain aux abords d'un cimetière, où les enfants font des tours de carrousel et les parents s'adonnent au "Schunkeln ", etc.

7 www.humba.de 
la relation entre lesdites " musiques du monde/Weltmusik " et les traditions musicales locales de la ville de Cologne, dont celle liée au carnaval est la plus puissante.

La première impulsion des activistes d' " Humba \& Famille " s'est manifestée lors d'un voyage en Côte d'Ivoire en 1992 pour une recherche musicale. Constatant, selon eux, que les locaux ne s'intéressaient guère à leur musique traditionnelle, qui était - et est - pourtant toujours la clé de nombreuses activités de musiciens à Cologne et en Afrique de l'Ouest, les activistes Humba s'interrogèrent : est-il acceptable de demander aux Africains de mieux cultiver leurs traditions musicales alors qu'en Allemagne une grande partie des personnes ignore les traditions musicales locales dans la plupart des régions?

Une première recherche a été menée sur les musiques du carnaval de Cologne et notamment les musiques traditionnelles jouées en ville. L'idée était de mieux comprendre les racines de certains courants musicaux, les nouvelles tendances passionnantes existant au-delà du folklore et FakeFolk ( " faux folklore "). II s'agissait aussi de saisir les effets produits par la diversité des musiques issues des migrations à la fois sur la circulation transnationale de la musique et sur la création locale.

La recherche a été soutenue par WDR Radio et a abouti à une présentation live lors du carnaval de 1994, sous le titre "Humba Party ", et à une première compilation en CD. Depuis, le mouvement " Humba \& Famille " a créé un environnement fertile pour l'expérimentation musicale. Les musiciens d'origines diverses sont pleinement reconnus comme des acteurs de l'expression musicale urbaine, et comme des interprètes de la musique de carnaval, permettant au public de danser, chanter ou rire.

Et comment cette fusion musicale à Cologne est-elle devenue une tradition populaire vivante et bien ancrée ? Pour certains artistes, ce fut aisé, car ils venaient d'une autre culture du carnaval, comme celle du Brésil, de Cuba, etc. ; mais d'autres musiciens ont dû transformer leur style traditionnel, soit musicalement ou en travaillant avec le dialecte kölsch, très important pour la culture locale et particulièrement pour le carnaval. Ils ont produit et créé des chansons de rap, de reggae, de samba ou des chansons orientales en kölsch et des Krätzcher dans d'autres langues. Mais au lieu de se tourner vers les genres comme le rock ou la pop anglophone, le mouvement Humba incarne l'âme de Cologne. Sa mission va bien au-delà du carnaval, car il veut libérer les traditions musicales locales des associations négatives avec l'époque nazie qui existaient encore dans les années 1990 et 2000, et transgresser les différences entre les classes sociales. À l'époque actuelle, de grands événements populaires comme "Loss' m'r singe " ("Allons chanter ") remplissent le plus grand stade de Cologne, où 40000 personnes se retrouvent pour chanter des chansons de Noël, jouées entre autres par les groupes du carnaval. Non seulement le carnaval dure toute l'année par le biais de ces prétextes pour se retrouver, faire la fête et chanter, mais il représente un état d'esprit qui permet de transgresser des règles pendant toute l'année.

Après le premier succès des fêtes de carnaval organisées par Humba, les fans de la musique du mouvement Humba ont apporté leurs savoirs à d'autres 
occasions, inspirés de la saison du carnaval. En 2001, les fans et les activistes ont lancé des petits festivals populaires d'été dans les jardins de banlieue, le "Humba-Schrebergarten-Tour ", présentant toujours au moins un groupe du style local de Cologne et un ensemble de musique d'ailleurs - tradition africaine, classique perse, gamelan indonésien, etc. - tous basés en ville ou dans la région.

Par la suite, les activités se sont étendues à d'autres aspects de la vie locale urbaine. Elles ont par exemple porté sur les musiques des Edelweißpiraten, un mouvement de résistance de la jeunesse sous la dictature nazie. À l'origine ce fut un mouvement musical très important à Cologne, qui a inspiré les militants $d^{\prime} H u m b a$ à entrer en contact avec les anciens Edelweißpiraten et à produire avec eux et d'autres groupes de musique un festival pour honorer la lutte anti-nazie. Depuis, le Edelweißpiraten-Festival ${ }^{8}$ est devenu une expression majeure du patrimoine immatériel urbain et de la diversité culturelle de Cologne.

\section{Valoriser la multiplicité sonore des communautés (trans)locales}

Une autre facette de cette vie locale urbaine aux croisements de traditions locales et migratoires qui a été explorée par Humba concerne la collaboration avec la communauté gitane de Cologne pour préserver et promouvoir les différents aspects de leur culture. Un travail similaire a été effectué auprès de la communauté sinti locale présente depuis plusieurs générations, et ce, malgré le fait que leurs membres ont souvent été victimes d'exclusion. Aujourd'hui, retournant un ancien stigmate, ils se disent fièrement "Zigeuner " et ont ainsi donné ce nom au festival "Rheinisches Zigeunerfestival " ${ }^{9}$. Ce festival offre des concerts autour des voitures à cheval et chars historiques, que les nazis avaient volés à la famille de Markus Reinhardt, des gitans basés à Cologne. Le festival regroupe également des communautés gitanes translocales, issues de nombreux pays, montrant à la fois sa diversité et son unité.

Toutes ces activités menées par Humba \& Famille sont des formes de « laboratoires publics " permettant d'expérimenter avec les sons traditionnels et nouveaux, et d'interagir avec les musicien-ne-s locaux et leurs amis venant du monde entier ${ }^{10}$.

En dehors du carnaval à proprement parler, il y a également des paysages sonores des soi-disant "Immis ", les nouveaux arrivants, les migrants et les réfugiés, qui représentent près de $40 \%$ de la population. À Cologne, il n'y a

\footnotetext{
8 www.edelweisspiratenfestival.de

9 www.zigeunerfestival.de

10 Un grand nombre de familles translocales de gitans ont leur base à Cologne, au point d'y enterrer leurs morts dans de grands caveaux familiaux. Sur certaines pierres tombales du grand cimetière de l'Ouest, "Westfriedhof ", des voitures à cheval ou de grosses berlines sont gravées pour rappeler le passé et le présent nomade. Les familles se retrouvent régulièrement au cimetière pour entretenir les caveaux, mais aussi pour passer du temps ensemble dans ces lieux qui ressemblent à de grands parcs avec des arbres centenaires. Ce cimetière, voisin du cimetière juif, comporte aussi un carré musulman (Observations de Monika Salzbrunn, Westfriedhof, 23-24/12/2019).
} 
pas un groupe de migrants majoritaire, mais de nombreuses communautés : descendants d'Allemands ayant grandi en Pologne, en Russie ou en Ukraine, exilés d'Iran et d'Afghanistan depuis les années 1990, travailleurs migrants en provenance d'Italie et de Grèce depuis les années 1960. Les Arméniens, Kurdes et Turcs représentent d'autres grands groupes culturels, ainsi que les Asiatiques, les Latinos et, depuis 2015, les Syriens, les Yézidis d'Irak et les migrant·e-s venus d'Afrique de l'Ouest et du Nord. Avec l'apport de nouveaux instruments et styles musicaux, la ville est perçue comme un des symboles du multiculturalisme tolérant, d'un point de vue du discours sur soi et d'un point de vue électoral ${ }^{11}$. Parmi ces nouvelles personnalités artistiques, certaines conservent leur musique traditionnelle dans leur nouveau lieu de résidence au bord du Rhin et préservent la dimension traditionnelle et/ou rituelle de leur musique, principalement jouée pour leur propre communauté.

D'autres se lancent plutôt dans la recherche d'une nouvelle maison musicale et participent à des groupes interculturels et des projets de "Brauchtum " (" coutumes ") : une pratique culturelle à la fois enracinée localement, mais aussi cosmopolite et progressiste. Des professions ou des catégories de population ont également leur porte-parole musical, comme, par exemple, Köbes Underground (Köbes : "garçon " dans les brasseries de Cologne).

Ainsi, tous ces protagonistes musicaux participent, avec leurs graines sonores propres, à ériger la ville de Cologne en une véritable tour de Babel, notamment lorsque se mettent en branle des mouvements sociaux tels I'EdelweißpiratenFestival, en mémoire de la résistance de Cologne pendant la dictature nazie, le Rheinisches Zigeunerfestival des Sinti et Roms locaux, ou le festival de Newroz, "Printemps des cultures " de la scène kurde et irano-kölsch.

\section{Références bibliographiques}

Cornelissen Georg (2019) Kölsch. Portrait einer Sprache, Köln, Greven Verlag.

Fuchs Peter, Schwering Max-Leo und Zöller Klaus (1972) Kölner Karneval. Zur Kulturgeschichte der Fastnacht, Band 1, Köln, Greven Verlag.

Salzbrunn Monika (2014) How diverse is Cologne Carnival? How migrants appropriate popular art spaces, Identities, 21 (1), pp. 92-106.

11 Pour une analyse des actions en faveur des sans-papiers pendant le carnaval de Cologne, voir Salzbrunn (2014). Depuis un siècle, le vote pour l'extrême droite est le plus faible dans la Rhénanie, notamment à Aix-la-Chapelle et Cologne. 